\title{
Identificação Adaptativa Supervisionada Utilizando Marca d'Água Digital
}

\author{
Mário Uliani Neto, Leandro de C. T. Gomes e João Marcos T. Romano
}

\begin{abstract}
Resumo-Apresentamos aqui um método de identificação adaptativa supervisionada baseada no uso de uma marca d'água como sinal de referência. Ao contrário de métodos adaptativos tradicionais, nos quais a comunicação é periodicamente interrompida para a transmissão de seqüências de treinamento, uma marca d'água é transmitida ininterruptamente através do sistema, juntamente com o sinal de informação. Através da comparação entre o sinal recebido e a marca d'água processada pelo filtro identificador, os coeficientes deste filtro são continuamente adaptados para estimar as características do sistema. Resultados de simulação são apresentados para ilustrar o método e demonstrar sua viabilidade.
\end{abstract}

Palavras-Chave-Filtragem adaptativa, identificação adaptativa, marca d'água digital, superimposed training.

Abstract-We present a method for supervised adaptive identification based on the use of a watermark as reference signal. In contrast to traditional adaptive methods, characterized by the periodic interruption of communication for the transmission of training sequences, a watermark is transmitted uninterruptedly through the system, along with the information signal. By comparing the received signal and the watermark processed by the identification filter, the coefficients of this filter are continuously adapted to estimate system characteristics. Simulation results are presented to illustrate the method and to demonstrate its viability.

Keywords - Adaptive filtering, adaptive identification, digital watermark, superimposed training.

\section{INTRODUÇÃO}

O problema de identificação consiste na obtenção de um modelo matemático de um sistema ou, mais especificamente, de um canal de comunicação. Métodos tradicionais de identificação adaptativa supervisionada usam seqüências de treinamento como sinal de referência. Este paradigma consiste em interromper periodicamente a transmissão de informação para transmitir uma seqüência de treinamento capaz de estimular os diversos aspectos da resposta do sistema. A mesma seqüência é empregada na excitação de um filtro identificador cujos coeficientes são ajustados através da comparação de sua saída com a saída do sistema analisado, obtendo-se assim um modelo do sistema. Exemplos de algoritmos que tradicionalmente utilizam a abordagem citada são o LMS e o RLS [1].

No cenário descrito, o sinal que carrega informação é interrompido durante a transmissão das seqüências de treinamento.

Mário Uliani Neto e Leandro de C. T. Gomes, Centro de Pesquisa e Desenvolvimento em Telecomunicações (CPqD), Rod. Campinas-MogiMirim (SP 340), km 118,5, CEP 13086-902, Campinas, SP, Brasil; Tel: (19) 3705-6724; e-mail: \{uliani, tgomes\} @cpqd.com.br.

João Marcos T. Romano, DSPCom - Lab. de Proc. de Sinais para Comunicações Móveis - Fac. de Eng. Elétrica e de Computação - Universidade Estadual de Campinas (Unicamp), Caixa Postal 6101, CEP 13083-970, Campinas, SP, Brasil; Tel.: (19) 3521-3703, Fax: (19) 3289-1395; e-mail: romano@decom.fee.unicamp.br.
Em aplicações de tempo real, estas interrupções podem ser indesejáveis ou até mesmo inaceitáveis. Uma alternativa para evitar-se o uso de seqüências de treinamento é recorrer às técnicas de identificação cega ou não-supervionada, que não contam com sinais de referência, baseando-se geralmente na análise de estatísticas de ordem superior do sinal. No entanto, tais técnicas podem apresentar alguns problemas relativos à convergência para mínimos locais ou, em alguns algoritmos, custo computacional superior ao das técnicas supervisionadas.

Tendo em vista as limitações das técnicas tradicionais de identificação expostas nos parágrafos anteriores, apresentamos um método de identificação baseado no uso de uma marca d'água digital como sinal de referência. A marca d'água é um sinal similar em natureza ao sinal de informação (dito hospedeiro) e é continuamente inserida neste último ao longo do tempo. Ambos os sinais são simultaneamente transmitidos através do sistema que se deseja modelar. Assim como uma sequiência de treinamento, a marca d'água é conhecida no receptor, sendo utilizada para estimar as características relevantes do sistema. Neste esquema, ambos os sinais (de marca d'água e hospedeiro) são transmitidos continuamente, de modo que não haja interrupções na comunicação e os coeficientes do modelo de identificação possam ser constantemente atualizados.

Para que não haja interferências entre a marca d'água e o sinal hospedeiro, estes sinais devem ser independentes entre si. Além disso, parte da potência do sinal total transmitido deve ser utilizada para a marca d'água, o que implica em uma redução da potência do sinal de informação. O sucesso desta abordagem dependerá de uma adequada escolha de potências, refletida na relação sinal-marca d'água (SWR).

Uma técnica similar à que propomos é conhecida como superimposed pilot training [2], [3]. Esta técnica baseia-se na sobreposição ao sinal de informação de uma seqüência de dados piloto conhecida, após a etapa de modulação de um sistema de comunicação. A sequiência piloto é detectada no receptor e usada para identificar o canal de transmissão. A principal diferença entre esta técnica e os métodos de marca d'água é o conceito de transparência que norteia estes últimos: a marca d'água deve ser construída de forma tal que, quando encarada como um ruído adicionado ao sinal hospedeiro, sua influência na detecção e/ou percepção do sinal transmitido seja insignificante caso sejam utilizados receptores legados (i.e. não preparados para detectar o sinal de marca d'água). Dependendo da natureza do sinal de informação, modelos perceptuais podem ser utilizados no transmissor para garantir a transparência da marca d'água. A preocupação com a transparência inexiste na técnica de superimposed training.

$\mathrm{O}$ artigo está estruturado como descrevemos a seguir. $\mathrm{Na}$ 
seção 2, é feita uma breve revisão de marca d'água digital utilizando o método de espalhamento espectral. Na seção 3, é introduzido o método de identificação adaptativa baseado em marca d'água, apresentando-se uma análise fundamentada nas equações de Wiener. $\mathrm{Na}$ seção 4, são discutidos alguns elementos resultantes da análise precedente, com o auxílio de simulações computacionais. Finalmente, a seção 5 traz algumas conclusões e perspectivas de trabalhos futuros.

\section{BREVE REVISÃo DE MARCA D’ÁGuA}

Um sistema de marca d'água pode ser modelado como um sistema de comunicação, no qual a informação é transmitida através de um canal [4], [5]. Nesta analogia, o sinal de informação (hospedeiro) é visto como ruído, enquanto a marca d'água carrega informação útil. Do ponto de vista do usuário, a relação se inverte: o sinal de informação carrega informação útil, enquanto a marca d'água é percebida como ruído.

As primeiras técnicas de marca d'água foram propostas em meados da década de 90 como uma solução potencial para o problema da pirataria de documentos multimídia, em particular áudio, imagens e vídeo [6], [7]. Estas técnicas consistem na inserção de uma marca, a marca d'água, no documento a proteger (sinal hospedeiro). A marca d'água contém dados que podem ser extraídos por um detector a partir do sinal marcado (sinal hospedeiro + marca d'água). Apesar da adição da marca d'água, as características perceptuais do documento multimídia devem ser preservadas (propriedade denominada transparência), o que pode ser conseguido através de modelos perceptuais.

Além da proteção de direitos autorais, existem diversas outras aplicações para métodos de marca d'água. Cada aplicação exige um método adaptado às suas particularidades. Neste trabalho, foi estudado o uso da marca d'água para identificação de sistemas, tendo sido empregada a técnica de espalhamento espectral. Esta técnica foi escolhida por produzir um sinal de marca d'água ortogonal ao sinal de informação, o que facilita a sua posterior detecção.

\section{A. Geração de Marca d'Água por Espalhamento Espectral}

A técnica de espalhamento espectral [4], [5] consiste na adição de uma sequiência pseudo-aleatória $m(n)$ (a marca d'água) ao sinal de informação $t(n)$, resultando no sinal marcado $s(n)$ :

$$
s(n)=t(n)+\alpha m(n)
$$

Em geral, a energia da marca d'água é várias vezes inferior à energia do sinal de informação. A marca d'água será ortogonal ao sinal de informação, possibilitando a detecção da marca através de correlação. $\mathrm{O}$ fator de escala $\alpha$ controla o nível de degradação perceptual e robustez da detecção.

\section{IDENTIFICAÇÃO SUPERVISIONADA BASEADA EM MARCA D'ÁGUA}

O objetivo das técnicas de identificação é obter um modelo de um sistema desconhecido, tendo em mãos um sinal que sofreu influência dos efeitos desse sistema. Para tanto, uma seqüência de treinamento pode ser utilizada. Esta seqüência é injetada periodicamente nas entradas do sistema e de um filtro identificador; através da comparação entre as respectivas saídas, podem-se ajustar os coeficientes do filtro de modo que este modele da melhor maneira possível o sistema a identificar.

Esta abordagem apresenta duas dificuldades importantes. Durante o período de treinamento, a transmissão de informação útil através do sistema deve ser interrompida, o que pode ser inaceitável em diversas aplicações. Além disso, se as características do sistema variam significativamente entre os intervalos de treinamento, o modelo poderá deixar de representar adequadamente o sistema. A solução dessas duas dificuldades é contraditória: enquanto a primeira indica a necessidade de que os períodos de treinamento sejam espaçados, a segunda implica em treinamentos realizados com a maior freqüência possível.

O método que apresentamos soluciona esses dois problemas simultaneamente. O treinamento é realizado por meio de uma marca d'água superposta ao sinal de informação e transmitida ininterruptamente. O sinal de informação nunca é interrompido e os coeficientes do modelo são constantemente atualizados, buscando manter o filtro identificador sempre o mais próximo possível do sistema.

\section{A. Descrição do Algoritmo}

O esquema clássico de identificação supervisionada utilizando sequiência de treinamento é apresentado na Fig. 1. Este método baseia-se na comparação da saída $y(n)$ do modelo com um sinal $x(n)$ de referência, produzindo o sinal de erro $e(n)$. Durante o período de treinamento, o sinal $s(n)$ transmitido através do sistema é idêntico ao sinal na entrada do modelo. Quando o modelo é adequadamente ajustado, o sinal de erro $e(n)$ é minimizado, implicando em um sinal $y(n)$ na saída do modelo o mais próximo possível do sinal $x(n)$ na saída do sistema conforme o critério estabelecido. Esta operação é executada periodicamente para rastrear possíveis variações do sistema.

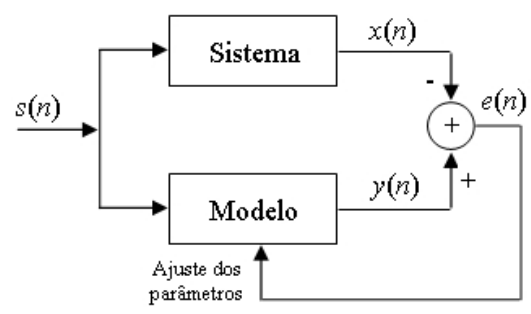

Fig. 1. Esquema clássico de identificação supervisionada

Uma medida comum para adequação do modelo é o erro quadrático médio (EQM), calculado a partir do sinal de erro $e(n)$, o que conduz a uma função custo $J$ que expressa o critério de Wiener:

$$
J=E\left[e(n) e^{*}(n)\right]=E\left[|e(n)|^{2}\right]
$$

onde $E$ denota o operador estatístico esperança. 


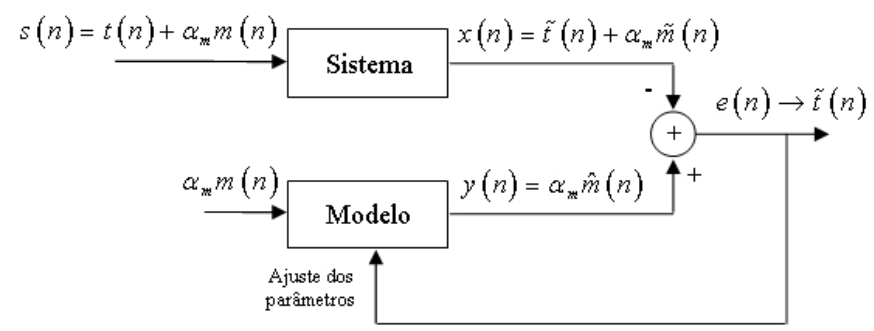

Fig. 2. Esquema de identificação utilizando marca d'água

A solução ótima para o problema de identificação é obtida através da minimização desta função custo com respeito aos coeficientes $\mathbf{w}$ do filtro modelo

$$
J_{\min }(\mathbf{w})=\min _{\mathbf{w}} E\left[|e(n)|^{2}\right]
$$

e os coeficientes ótimos $\mathbf{w}_{0}$ do modelo são dados por:

$$
\mathbf{w}_{o}=\arg \left(\min _{\mathbf{w}} E\left[|e(n)|^{2}\right]\right)
$$

Em nosso método, ao invés de substituir periodicamente o sinal de informação pela seqüência de treinamento, transmitimos constantemente uma marca d'água sobreposta ao longo do sinal de informação. A marca d'água é gerada a partir de uma seqüência pseudo-aleatória e é descorrelacionada com o sinal de informação $t(n)$. Obtém-se então o sinal de referência na saída do sistema:

$$
d(n)=x(n)=\tilde{t}(n)+\alpha_{m} \tilde{m}(n)
$$

Assumindo-se um sistema linear, o sinal $x(n)$ na saída do sistema pode ser visto como a soma dos sinais $\tilde{t}(n)$ e $\alpha_{m} \tilde{m}(n)$, onde $\tilde{t}(n)$ e $\tilde{m}(n)$ são, respectivamente, versões do sinal de informação e da marca d'água distorcidas pelo sistema. De forma similar, após a convergência dos coeficientes do modelo, a saída $y(n)$ do modelo linear pode ser vista como uma estimativa do sinal $\tilde{m}(n)$. Este esquema é ilustrado na Fig. 2. O sinal de erro $e(n)$ é expresso, neste caso, como:

$$
e(n)=y(n)-x(n)=\alpha_{m}(\hat{m}(n)-\tilde{m}(n))-\tilde{t}(n)
$$

A função custo $J$, definida como a esperança do quadrado do sinal de erro $e(n)$, será dada por $^{1}$ :

$$
J=E\left[(e(n))^{2}\right]=E\left[\left(\alpha_{m}(\hat{m}(n)-\tilde{m}(n))-\tilde{t}(n)\right)^{2}\right]
$$

A equação (7) pode ser reescrita em notação matricial. Define-se $\mathbf{m}(n)$ e $\mathbf{t}(n)$ como $^{2}$ :

$$
\begin{gathered}
\mathbf{m}(n)=[m(n), m(n-1), \ldots, m(n-M+1)]^{T} \\
\mathbf{t}(n)=[t(n), t(n-1), \ldots, t(n-M+1)]^{T}
\end{gathered}
$$

\footnotetext{
${ }^{1}$ Por simplicidade, a análise apresentada tem por base sinais reais, embora possa ser estendida a sinais complexos.

${ }^{2}$ Caracteres em negrito são usados aqui para denotar grandezas vetoriais e matriciais
}

Os vetores $\mathbf{m}(n)$ e $\mathbf{t}(n)$ têm comprimento $M$, onde $M$ expressa o número de coeficientes do modelo.

Os sinais $x(n)$ e $y(n)$ podem ser representados através da convolução dos sinais de entrada do sistema e do modelo com as respectivas funções de transferência:

$$
\begin{gathered}
x(n)=\mathbf{h}^{T}\left(\mathbf{t}(n)+\alpha_{m} \mathbf{m}(n)\right) \\
y(n)=\alpha_{m} \mathbf{w}^{T} \mathbf{m}(n)
\end{gathered}
$$

onde $\mathbf{h}=\left[h_{0} \cdots h_{L-1}\right]$ expressa os coeficientes do sistema FIR com função de transferência de comprimento $L$ e $\mathbf{w}=$ $\left[w_{0} \cdots w_{M-1}\right]$ representa os coeficientes do modelo FIR de comprimento $M$. Com essas definições, o sinal de erro pode ser representado da seguinte forma:

$e(n)=y(n)-x(n)=\alpha_{m} \mathbf{w}^{T} \mathbf{m}(n)-\mathbf{h}^{T}\left(\mathbf{t}(n)+\alpha_{m} \mathbf{m}(n)\right)$

Ao se determinar a esperança do erro quadrático, conforme o critério estabelecido, chegamos à expressão:

$$
\begin{aligned}
& J(\mathbf{w})=E\left[(x(n))^{2}\right]-\mathbf{w}^{T} E\left[\alpha_{m}^{2} \mathbf{m}(n) \mathbf{h}^{T} \mathbf{m}(n)\right]- \\
& E\left[\alpha_{m}^{2} \mathbf{h}^{T} \mathbf{m}(n) \mathbf{m}^{T}(n)\right] \mathbf{w}+\mathbf{w}^{T} E\left[\alpha_{m}^{2} \mathbf{m}(n) \mathbf{m}^{T}(n)\right] \mathbf{w}
\end{aligned}
$$

Em decorrência da técnica de espalhamento espectral empregada para a geração da marca d'água, os sinais $t(n)$ e $\alpha_{m} m(n)$ são independentes entre si. Portanto, podemos definir a relação:

$$
\alpha_{m} \tilde{m}(n)=\alpha_{m} \mathbf{h}^{T} \mathbf{m}(n)
$$

Analisando a equação (13) e de posse da relação expressa em (14), chegamos às seguintes definições:

$$
\begin{aligned}
& E\left[(x(n))^{2}\right]=\sigma_{x}^{2} \\
& E\left[\alpha_{m}^{2} \mathbf{m}(n) \mathbf{h}^{T} \mathbf{m}(n)\right]=E\left[\alpha_{m} \mathbf{m}(n) \tilde{m}^{*}(n)\right]=\mathbf{p}_{m} \\
& E\left[\alpha_{m}^{2} \mathbf{m}(n) \mathbf{m}^{T}(n)\right]=\mathbf{R}_{m}
\end{aligned}
$$

onde assume-se $t(n)$ com média zero.

Substituindo essas relações na equação (13), obtemos a superfície de erro do critério de Wiener na forma canônica para o sistema de identificação utilizando marca d'água:

$$
J(\mathbf{w})=\sigma_{x}^{2}-\mathbf{w}^{T} \mathbf{p}_{m}-\mathbf{p}_{m}^{T} \mathbf{w}+\mathbf{w}^{T} \mathbf{R}_{m} \mathbf{w}
$$

Para obtermos o ponto de mínimo da função custo, é necessário minimizar a função $J(\mathbf{w})$ expressa em (16) com respeito aos coeficientes $\mathbf{w}$ do modelo. Como $J(\mathbf{w})$ expressa um quadrado perfeito, é possível reescrever (16) da seguinte forma:

$$
J(\mathbf{w})=\sigma_{x}^{2}-\mathbf{p}_{m}^{T} \mathbf{R}_{m}^{-1} \mathbf{p}_{m}+\left(\mathbf{w}-\mathbf{R}_{m}^{-1} \mathbf{p}_{m}\right)^{T} \mathbf{R}_{m}\left(\mathbf{w}-\mathbf{R}_{m}^{-1} \mathbf{p}_{m}\right)
$$


Para atingir o ponto de mínimo da equação (17), o termo $\left(\mathbf{w}-\mathbf{R}_{m}^{-1} \mathbf{p}_{m}\right)$ deve igualar-se a zero. Portanto, para minimi$\operatorname{zar} J(\mathbf{n})$ :

$$
\mathbf{w}_{0}-\mathbf{R}_{m}^{-1} \mathbf{p}_{m}=0 \quad \Rightarrow \quad \mathbf{w}_{0}=\mathbf{R}_{m}^{-1} \mathbf{p}_{m}
$$

Com o ponto de mínimo expresso em (18), obtemos o valor mínimo da função custo:

$$
\min _{\mathbf{w}} J(\mathbf{w})=\sigma_{x}^{2}-\mathbf{p}_{m}^{T} \mathbf{R}_{m}^{-1} \mathbf{p}_{m}
$$

Os termos $\mathbf{R}_{m}$ e $\mathbf{p}_{m}$ expressam, respectivamente, a autocorrelação e a correlação-cruzada do sinal da marca d'água. O ponto de mínimo obtido no esquema de identificação utilizando marca d'água, mostrado na equação (18), depende apenas das características do sinal de marca d'água, bastando o conhecimento deste sinal para que seja possível o ajuste dos coeficientes do modelo. Isso significa que, independentemente das características do sinal de informação, o filtro utilizado como modelo convergirá para um ponto que representa tão bem quanto possível o sistema segundo o critério de Wiener. Demonstra-se assim a viabilidade do uso da marca d'água em identificação supervisionada.

Nota-se em (16) e (19) que apenas a superfície de erro sofrerá a influência do sinal de informação, apresentando um deslocamento no sentido do custo $J$, representado por $\sigma_{x}^{2}$ (variância do sinal $x(n)$ ). Porém, como demonstrado, esse deslocamento não influenciará o sentido dos coeficientes $\mathbf{w}$.

Diferentemente do caso de identificação, foi demonstrado em [9] que o ponto de convergência em um sistema de equalização utilizando marca d'água é dependente do sinal de informação, fazendo-se necessária a utilização de um método de branqueamento quando o sinal de informação é correlacionado.

\section{RESUltados E Discussão}

Nas simulações, utilizamos o algoritmo RLS para a adaptação do modelo. Inicialmente, o canal foi modelado como um filtro linear com função de transferência $\mathrm{H}_{1}(z)=$ $1-0.6 z^{-1}$. As amostras do sinal transmitido através do sistema são i.i.d. e pertencentes a uma constelação binária 2-PAM ou 2-PSK de módulo unitário. Supõe-se que não há ruído aditivo. O modelo de identificação contou com o mesmo número de parâmetros do sistema, e seus coeficientes foram inicializados na origem.

A figura 3 apresenta a evolução temporal dos coeficientes do modelo para uma realização do algoritmo RLS com SWR $=0 \mathrm{~dB}$ e fator de esquecimento $\lambda=1$. A Fig. 4 ilustra a realização do algoritmo RLS para $\mathrm{SWR}=20 \mathrm{~dB}$, que é o limite aproximado de potência para o qual sistemas de marca d'água baseados em modelos perceptuais geralmente garantem a transparência da marca [4]. Nota-se que, em ambos os casos, o algoritmo RLS atingiu rapidamente os pontos de mínimo.

É possível mostrar [1] que o desvio quadrático médio do algoritmo RLS é definido por:

$$
\mathcal{D}(n)=\frac{1}{n} \sigma_{o}^{2} \sum_{i=1}^{M} \frac{1}{\lambda_{i}}, \quad n>M
$$

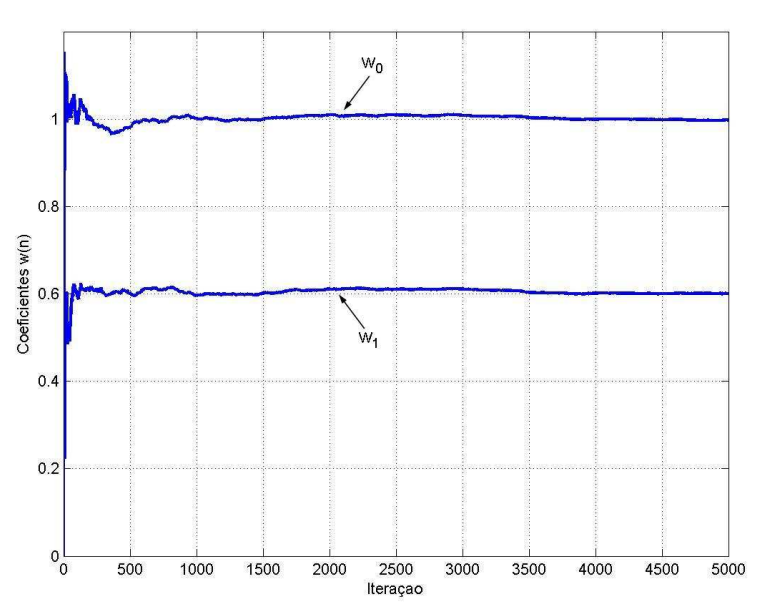

Fig. 3. Evolução temporal do algoritmo RLS para $S W R=0 \mathrm{~dB}$

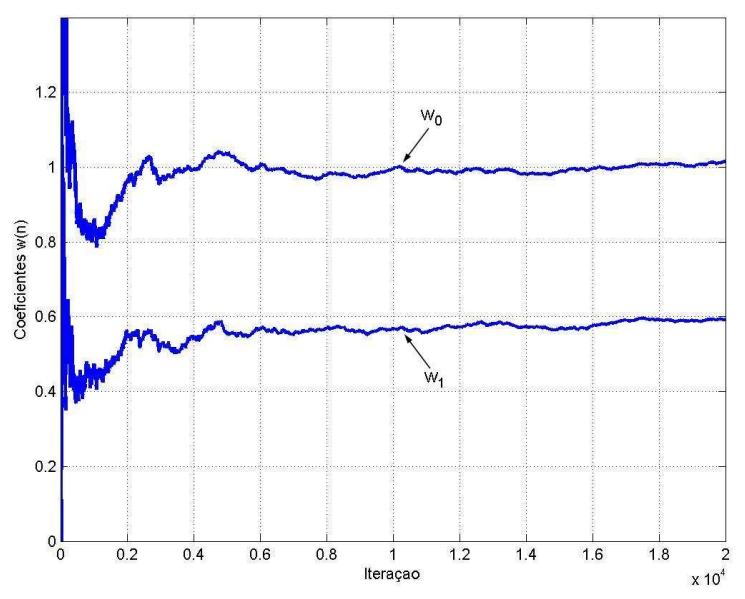

Fig. 4. Evolução temporal do algoritmo RLS para SWR $=20 \mathrm{~dB}$

onde $\lambda_{i}$ são os autovalores da matriz de correlação $\mathbf{R}_{m}, \sigma_{o}^{2}$ é a variância de $e_{o}(n)$ e $M$ expressa o comprimento do filtro modelo.

No esquema proposto, os autovalores da matriz de correlação $\mathbf{R}_{m}$ dependem apenas do sinal de marca d'água. Sendo assim, ao considerar-se um baixo número de iterações $n$, o desvio quadrático médio cresce à medida que diminui a potência da marca d'água, pois diminuem os valores de $\lambda_{i}$. Isto justifica o fato de as oscilações nas iterações com baixo valor de $n$ aumentarem à medida que diminui a potência da marca. No entanto, o desvio quadrático médio $\mathcal{D}$ decai quase linearmente à medida que aumenta o número de iterações $n$. Assim, os coeficientes estimados pelo algoritmo convergem na média (i.e. pelo critério de erro quadrático) para o vetor de parâmetros $\mathbf{w}_{o}$ quase linearmente em função do tempo.

Também é possível mostrar [1] que o algoritmo RLS não apresenta desajuste dos coeficientes na média. $\mathrm{O}$ valor médio de convergência do algoritmo RLS pode ser escrito como:

$$
E[\hat{\mathbf{w}}(n)] \approx \mathbf{w}_{o}-\frac{\delta}{n} \mathbf{p}_{m}, \quad n>M
$$


onde $\mathbf{p}_{m}$ é o vetor de correlação cruzada e $\delta$ uma constante positiva. Nota-se que o algoritmo RLS converge para o valor médio e é invariante com relação ao autovalor da matriz de correlação $\mathbf{R}_{m}$. Para um valor finito de $n$ maior do que $M$, a estimativa $\hat{\mathbf{w}}$ apresenta um desvio dependente da inicialização do algoritmo $\boldsymbol{\Phi}(0)=\delta \mathbf{I}$; porém, este desvio tende a zero conforme aumenta o número de iterações $n$. Dessa forma, o erro quadrático médio em excesso do algoritmo RLS converge a zero com um número de iterações $n$ tendendo ao infinito.

Foram realizadas também simulações com um sistema de fase não-mínima modelado como um filtro linear com função de transferência $\mathrm{H}_{2}(z)=1+1.2 z^{-1}-0.3 z^{-2}+0.8 z^{-3}$. O ensaio foi dividido em duas etapas. A primeira etapa utilizou um sinal transmitido formado por amostras i.i.d. extraídas de uma constelação 2-PAM ou 2-PSK de módulo unitário. A segunda etapa utilizou um sinal fortemente correlacionado, com amostras de áudio discreto (música pop). Em ambos os casos, desconsideramos o ruído e tomamos um modelo de identificação com o mesmo número de parâmetros do sistema, inicializados na origem. Para avaliar de maneira mais precisa o desempenho do método, definimos a seguinte medida de desvio em relação em relação ao caso ideal, proposta e discutida em [10]:

$$
\mathrm{D}=\frac{\left\|\mathbf{h}_{\text {obtido }}-\mathbf{h}\right\|}{\|\mathbf{h}\|}
$$

sendo $\mathbf{h}_{\text {obtido }}$ a estimativa do vetor de parâmetros e $\mathbf{h}$ seu valor exato.

No primeiro ensaio, utilizando amostras i.i.d., obtivemos os valores médios apresentados na tabela I. Para ambos os valores de SWR, o valor médio de $\mathrm{D}$ revela um grau de precisão satisfatório.

TABELA I

DESVIO DO ALGORITMO RLS COM SINAL I.I.D.

\begin{tabular}{|c|c|}
\hline \hline$S W R$ & Valor D \\
\hline \hline $20 d B$ & 0.000473 \\
\hline $23 d B$ & 0.000562 \\
\hline
\end{tabular}

No ensaio utilizando amostras discretas de áudio, obtivemos os valores apresentados na tabela II. Neste caso, o valor médio de D apresenta um patamar que também consideramos satisfatório.

\section{TABELA II}

DESVIO DO ALGORITMO RLS COM SINAL DE ÁUDIO

\begin{tabular}{|c|c|}
\hline \hline$S W R$ & Valor $D$ \\
\hline \hline $20 d B$ & 0.000634 \\
\hline $23 d B$ & 0.001082 \\
\hline
\end{tabular}

O algoritmo LMS também foi utilizado em testes. No entanto, este algoritmo necessita de uma quantidade muitas vezes maior de amostras para convergir se comparado ao RLS na faixa de SWR = $20 \mathrm{~dB}$. Essa característica pode ser demonstrada supondo a curva de convergência do algoritmo LMS aproximada por uma exponencial com constante de tempo $(\tau)_{E Q M, m e}$. Pode-se definir a seguinte constante de tempo média para o algoritmo [1]:

$$
(\tau)_{E Q M, m e} \approx \frac{1}{2 \mu \lambda_{m e}}
$$

onde $\mu$ é o passo de adaptação e $\lambda_{m e}$ define um autovalor médio para a matriz de autocorrelação $\mathbf{R}_{m}$ :

$$
\lambda_{m e}=\frac{1}{M} \sum_{i=1}^{M} \lambda_{i}
$$

onde $\lambda_{i}, i=1,2, \ldots, M$, são os autovalores da matriz de correlação $\mathbf{R}_{m}$ e $M$ expressa o comprimento do filtro modelo.

\section{Conclusões}

Neste trabalho, analisamos uma nova proposta de identificação de sistemas supervisionados utilizando uma marca d'água como sinal de referência. $\mathrm{O}$ método apresentado adiciona uma marca d'água ao sinal de informação e, sem interrupções na transmissão deste último, vale-se continuamente do sinal da marca d'água no filtro identificador para obter um modelo matemático do sistema.

O método proposto apresenta algumas importantes vantagens em relação às técnicas tradicionais de identificação. Dentre elas, podemos destacar a ausência de interrupções periódicas do sinal de informação para transmissão de seqüências de treinamento, ao mesmo tempo em que se evita a complexidade de critérios não-supervisionados ou cegos. Além disso, a técnica proposta tem uma ampla gama de aplicações, desde a identificação de canais de comunicação digital até o uso em sistemas de áudio e voz.

As simulações realizadas demonstram que o método pode trazer ganhos de desempenho em sistemas de comunicação, principalmente em situações nas quais a escassez de recursos sistêmicos inviabiliza o uso de técnicas tradicionais de identificação.

Nas próximas etapas deste trabalho, serão estudadas técnicas de ressincronização que aumentarão a robustez da detecção da marca d'água. Além disso, será pesquisado o uso de modelos psico-acústicos para aumentar a energia da marca d'água sem introduzir degradação perceptível em aplicações de áudio e voz.

\section{REFERÊNCIAS}

[1] S. Haykin, Adaptive filter theory, third edition, Prentice Hall, 1996.

[2] A. R. Varma, L. L. H. Andrew, C. R. N. Athaudage, J. H. Manton, Iterative algorithms for channel identification using superimposed pilots, Australian Communications Theory Workshop, February, 2004.

[3] J. K. Tugnait, and W. Luo, On channel estimation using superimposed training and first-order statistics, IEEE Communications Letters, vol. 7, no. 9, September, 2003.

[4] L. de C. T. Gomes, P. Cano, E. Gómez, M. Bonnet, E. Batlle, Audio watermarking and fingerprint: for which applications?, Journal of New Music Research, vol. 32, 2003.

[5] L. de C. T. Gomes, E. Gómez, N. Moreau, Resynchronization methods for audio watermarking, Proceedings of 111th AES Convention, New York, USA, November, 2001.

[6] H. J. Kim, Audio watermarking techniques, Pacific Rim Workshop on Digital Steganography, Kyushu Institute of Technology, Kitakyushu, Japan, July, 2003.

[7] P. Moulin, and R. Koetter, Data-hiding codes, Proceedings of the IEEE, vol. 93, no. 12, December, 2005.

[8] L. de C. T. Gomes, M. Mboup, M. Bonnet, Cyclostationarity-based audio watermarking with private hidden data, 109th Convention of Audio Engineering Society, Los Angeles, September, 2000. 
XXV SIMPÓSIO BRASILEIRO DE TELECOMUNICAÇÕES - SBrT 2007, 03-06 DE SETEMBRO DE 2007, RECIFE, PE

[9] M. Uliani Neto, Leandro de C. T. Gomes, João Marcos T. Romano, and Madeleine Bonnet, Adaptive equalization based on watermarking, VI International Telecommunications Symposium (ITS2006), September, Fortaleza, Brazil, 2006.

[10] J. Arenas García, V. Gómez Verdejo, M. Martínez Ramón, A. R. Figueiras Vidal, Separate-Variable Adaptive Combination of LMS Adaptive Filters for Plant Identification, IEEE, XIII Workshop on Neural Networks for Signal Processing, 2003. 